\title{
Auditory-Tactile Interaction Using Digital Signal Processing In Musical Instruments
}

\author{
Parul Chauhan \\ Manipal Institute of Technology, Manipal - 576104 (Karnataka State), INDIA
}

\begin{abstract}
This paper lays emphasis on research that gives an insight to the auditory-tactile perception in electronic musical instruments (here, electronic violin) stating that most traditional musical instruments inherently convey tactile feedback to the performer along with the auditory feedback which leads to a tight performer-instrument relationship which is not found in electronic musical instruments. Hence, introducing the phenomenon of coupled perception of sound and vibration using digital signal processing in order to facilitate better auditory perception in electronic instruments. Thus the main objective of study is Audio-Driven Vibration Feedback for auditory perception by the violinists in an Electronic Violin. The expected results of the research project ((here electronic violin) are supposed to be claimed beneficial for introducing the phenomenon of coupled perception of sound and vibration in order to facilitate better auditory perception in electronic instruments.
\end{abstract}

Keywords: Amplitude modulation, Audio-Driven Vibration Feedback, Digital Signal Processing, Octave Shift, Vibrotactile.

\section{INTRODUCTION}

The scope of the study is the Audio-Driven Vibration Feedback for auditory perception by the violinists in an Electric Violin, focussing on the audio tactile with an effect of integrated vibrotactile feedback on the "sensation" of an electronic musical instrument. Most traditional musical instruments convey a hint of tactile feedback to performers in addition to the auditory feedback. While the majority of the electronic instruments provide only auditory feedback to the performer which, eventually, results in less sense of instrument's response to the players' gestures than is available in the traditional instruments. [1] This integration of vibrotactile feedback into the design of the instrument is embodied using digital signal processing approach with the help of real-time analysis through Pure data, leading to the development of a wide variety of audio tactile algorithms based on a wide range of physical properties, providing a better perception, thus underlining the increasing importance of the performance acoustics.

\subsection{Motivation}

"Audio" has been vital to the development of musical skill for millennia, but the recent dominance of electronic technology in composition and sound production techniques has separated embodiment from experiences of playing. Electronic music culture needs to be compensated for this missing perceptual element in some or the other way. It is well known that acoustic vibrations are utilized for self-monitoring in acoustic performance, and that vibrotactile feedback can considerably improve touch perception without adding significant complexity or cost. This combination of circumstances should make integration of vibrotactile feedback systems into electronic musical instruments a major priority for instrument designers. The development of various techniques for displaying artificial vibrations in the frequency and amplitude ranges of musical sound is noticeable because vibrotactile feedback may improve controllability of certain musical processes.[7]

Most of the previous research work in reference to violin has limited contribution to the field of vibrotactile feedback for perceptual analysis. [2 34 4] The realisation of the missing link between the (electronic) sound source and its 'feel' leads to more development of the importance of the vibrational perception in electronic instruments.Unlike acoustical instruments, electronic musical instruments display a synthetic sound source which in most cases only can be heard and not felt. The non-auditory information is thought to be used by the player to improve articulation in producing the sound. Hence, the study emphasis on development of different signal processing approaches using Pure Data to generate suitable perceivable vibrations from sound.

\section{Methodology}

With most instruments, the player is in direct contact with the vibrating parts. For example, here the violin player is able to feel the string vibrating with the fingers of the left hand, and the player feels the vibration in the chin holder. It is known that the vibration amplitudes are well above threshold levels of tactile perception. 
Hence, of the different types of mechanoreceptors in the skin, the Pacinian corpuscles play the largest role in our vibrotactile perception because of their sensitivity in the audible range. Electronic sound sources are able to produce many different sounds, but each sound has the same 'feel' because nothing physically changes in the control device. In the real world, for example, a thicker string will produce a lower, heavier sound and gives more force feedback. Vibrational feedback has to be forced in the instrument so as to obtain the desirable vibration perceptions. [5]

The vibration signal is used as a sign carrier. It is pointing at a specific audio sequence from which it was generated. The goal is to identify a specific audio loop (e.g., a rattling shaker) using touch feedback. The acoustical signal needs to be abstracted and converted into a suitable vibration signal. In an instrument it is beneficial for the performer to feel the vibrations synchronous to the sound production mechanism. If we wish to provide vibrations in an electronic instrument (here violin), these vibrations must then be directly linked to the sound production. Such a link can be achieved by deriving the vibrotactile feedback signal.

The term electronic musical instrument is generally used to describe musical instruments in which the control interface and sound synthesis functions are physically separable, and which involve the transmission of digital control data from one to the other. Performer actions are usually detected and measured using a variety of sensing technologies, sampled and processed before being mapped to the control of some physical model or sound synthesis parameter. In addition to sound, non-audio feedback channels may be generated by software and sent back to the performer via their interface. This feedback here includes vibrations in the system displayed with various modalities using digital signal processing.

When producing vibrotactile feedback it is interesting to note that neither the actuators used to produce vibrotactile feedback or the human skin offer a response to vibrations across the frequency range. By having separate control over the frequency content of the signal sent to the vibrotactile feedback system we can compensate for these responses.[1] Thus by modifying the signal through pure data, suitable audio signal is sent to the loudspeakers so as to obtain suitable perceivable response.

In order to physically produce these vibrations, an actuator are needed which meets the following requirements:

1) It must be capable of producing the full frequency range of human tactile sensation.

2) It must be able to offer independent control of frequency, amplitude and waveform.

3) It must allow a large range of amplitude control (to allow for instrument dynamics).

4) It can be driven by an audio signal or a signal easily derived from an audio signal.

Previous studies show that voice-coil, the tactor and the piezoelectric element each of them meet the requirements to different extents. Of these, the voice coil offers the greatest range of frequency and amplitude control. Also of interest is that if we use a voice coil in the form of a loudspeaker, then the system can also be used as the main sound production method of the instrument. This not only adds sound-related vibrotactile feedback to the instrument but also co-locates the sound production into the instrument itself.

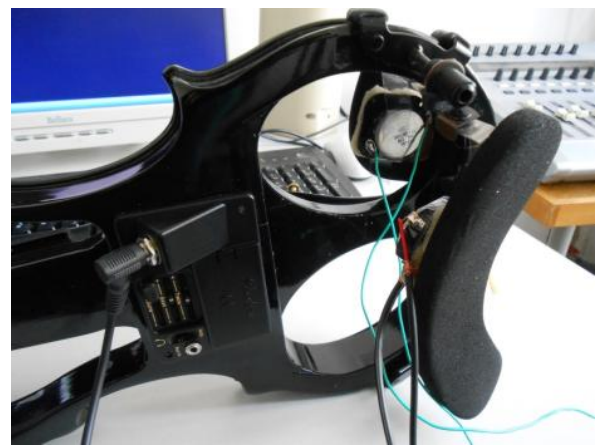

Fig.1. Hardware Setup of Feedback actuators to the electronic violin

\section{Algorithm In Order To Obtain Suitable Perceivable Vibrations}

Two different approaches to modify the frequency content of the audio signal (using digital signal processing) in order to obtain suitable perceivable vibrations are discussed. [6] 


\subsection{Amplitude modulation approach:}

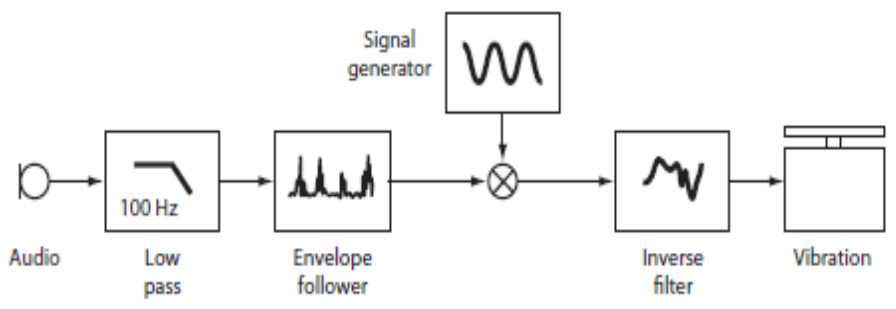

Fig.2. Block diagram for Amplitude Modulation approach

Relatively lower frequencies are more perceivable than the higher frequencies facilitating better sensation of vibrations produced, thus we need to extract the lower frequency contents. With the help of pure data, low pass-filtered at $100 \mathrm{~Hz}$, was included. The input signal from the violin is multiplied with the envelope of the original low-pass-filtered signal to retain the timing information. An envelope follower calculating the RMS amplitude of the input signal using successive analysis windows (Hanning windowing), with the window size set to 1024 samples, which corresponded to approximately $21 \mathrm{~ms}$, to avoid smearing the impulsive signal content. The period for successive analysis was half the window size.

\subsection{Octave Shift Approach:}

Another approach to obtain perceivable vibration is to shift down the frequency of all signals, thus decreasing the higher frequencies to a range of perceivable vibration sensation. With regard to sampling we can change the speed of an existing sound in an array, also resulting in the change in pitch. Using granular synthesis we can decouple these parameters. The concept of granular synthesis is that a sound is sampled at the original speed, but it is played at a different speed from each sample point.

In order to maintain partials, low-pass-filtered signals were used as a starting point. Hanning window is used to suppress the clicks that result with every transition to a new value, and a low pass filter to avoid high frequency artefacts that are not felt anyway. Using this method, some high-frequency artefacts occurred, which were filtered afterward by an additional low-pass filter at $100 \mathrm{~Hz}$.

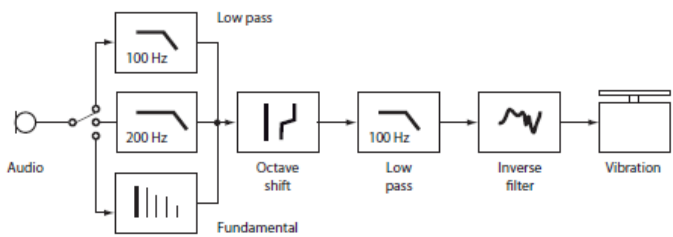

Fig. 3. Block diagram for Octave Shift Approach

Therefore, in this study the decision was made to shift all frequencies down one octave relative to their original frequency, with stronger compression toward higher frequencies. Again, Pd was used for pitch-shifting using a granular synthesis approach. The signal was divided into grains of 1000 samples, which were slowed down by half and summed again.

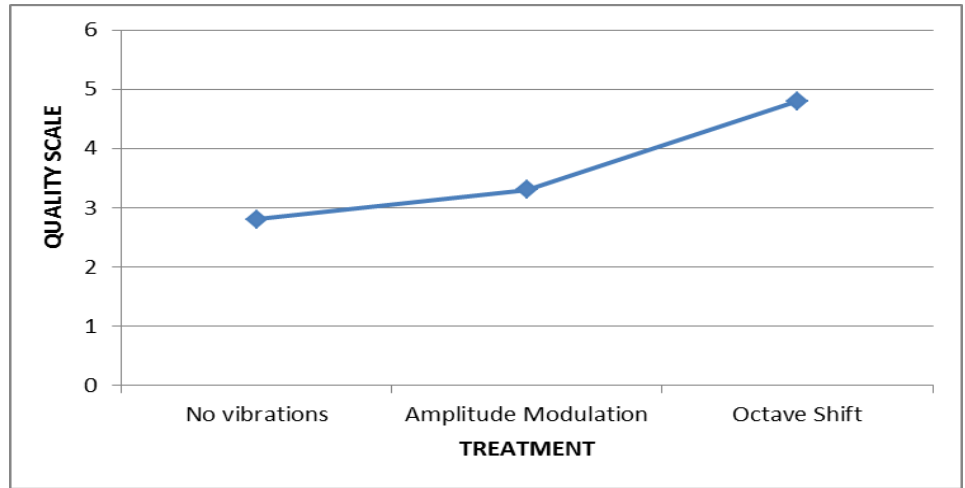

Fig. 4 Graphical representation of quality analysis of Audio-Driven Vibration Feedback 
As shown in the graphical representation of the quality analysis by considering the average value of ratings given by 6 participants on the quality scale which are interpreted as numbers on a linear scale from 1 to 5 , with 1 corresponding to 'bad' and 5 to 'excellent'. The quality improves by introducing vibrations in comparison to without vibrations. Also shows that the Octave Shift method is more effective than Amplitude Modulation Techniques. The reasons for this analysis could possibly be the string resonance affecting the violin quality. As the string resonates and excite resonance in the violin body at low frequencies, and the main bridge resonance at higher frequencies. Thus feedback introduces some extra unrequired vibrational components in the body of violin. Hence, Octave Shift technique is considered better in comparison with the Amplitude Modulation technique.

\subsection{Method of Investigation}

\section{Result Analysis}

AttrakDiff ${ }^{\mathrm{TM}}$ is used as an instrument for measuring the interactive quality, with the help of which the users can indicate their perception of the product. The average values of the AttrakDiff ${ }^{\mathrm{TM}}$ dimensions for the evaluated product are plotted on the diagram. In this presentation the hedonic quantity distinguishes between the aspects of stimulation and identity. Furthermore, the rating of attractiveness is presented.[8 9]

\begin{tabular}{|l|l|}
\hline Product title of fore-study & Without tactile feedback \\
\hline Product title of after-study & With tactile feedback \\
\hline Evaluation type & Comparison before-after, i.e. the project is rated twice. \\
\hline Variant & The same test participants in both project parts \\
\hline No. of Ratings in Fore-Study & 6 \\
\hline No. of Ratings in After Study & 6 \\
\hline
\end{tabular}

Table 1. Characteristics of Investigation

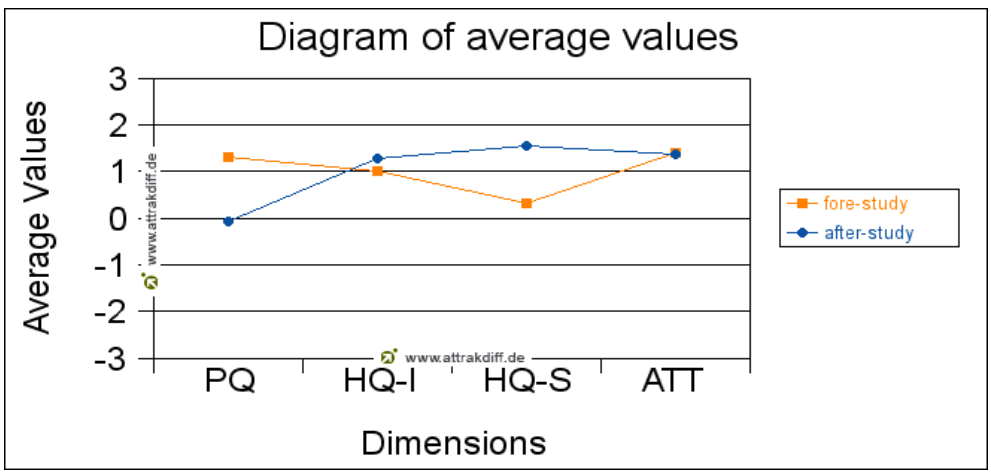

Fig 5. Mean values of the four AttrakDiff ${ }^{\mathrm{TM}}$ Dimensions for the product in the fore-study ("without tactile feedback") and after-study ("with tactile feedback")

\subsection{Interpretations of the study}

\subsubsection{Project part fore-study, product "without tactile feedback".} standards.

In terms of pragmatic quality the product is located in the above -average region. It meets ordinary Result: Must strive to improve the quality even more.

With regard to hedonic quality-identity, the product is located in the average region; it provides the user with identification.

\section{Result: Scope of improvement.}

With regard to hedonic quality-- stimulation, the product is located in the average region.

Result: In order to motivate, enthral and stimulate users even more intensely, possibility to aim for further improvement.

The products attractiveness value is located in the above-average region.

Result: the overall impression of the product is very attractive. 


\subsubsection{Project part after-study "with tactile feedback".}

In terms of pragmatic quality the product is located in the above-average region.

\section{Result: should you Result: Possibility of further improvement.}

With regard to hedonic quality identity the product is located in the above average region; it provides the user with the identification.

\section{Result: Possibility of further improvement.}

With regard to the hedonic quality stimulator the product is located in the above average region. It stimulates users awakens curiosity and motivates them.

\section{Result: In terms of aspects of stimulation the product is classified optimal}

The products attractiveness value is located in the above average region.

Result: the overall impression of the product is very attractive.

\subsection{Comparison of results of both project parts:}

In terms of pragmatic quality, the results have deteriorated in comparison to that of the fore-study. This difference is however statistically insignificant.

In terms of the identity aspect of hedonic quality, the results have improved in comparison to that of the fore-study. This difference is however statistically insignificant.

In terms of the stimulation aspect of hedonic quality, the product has improved in comparison to that of the fore-study. This difference is statistically significant.

In terms of overall, the product has improved in comparison to that of the fore-study. This difference is however statistically significant.

\begin{tabular}{|l|l|}
\hline Age & $20-40$ \\
\hline Number of Participants & 6 \\
\hline Gender & Male: 4 Female:2 \\
\hline Product experience & More than 3 years. \\
\hline
\end{tabular}

Table 2. Classification of Test Participants

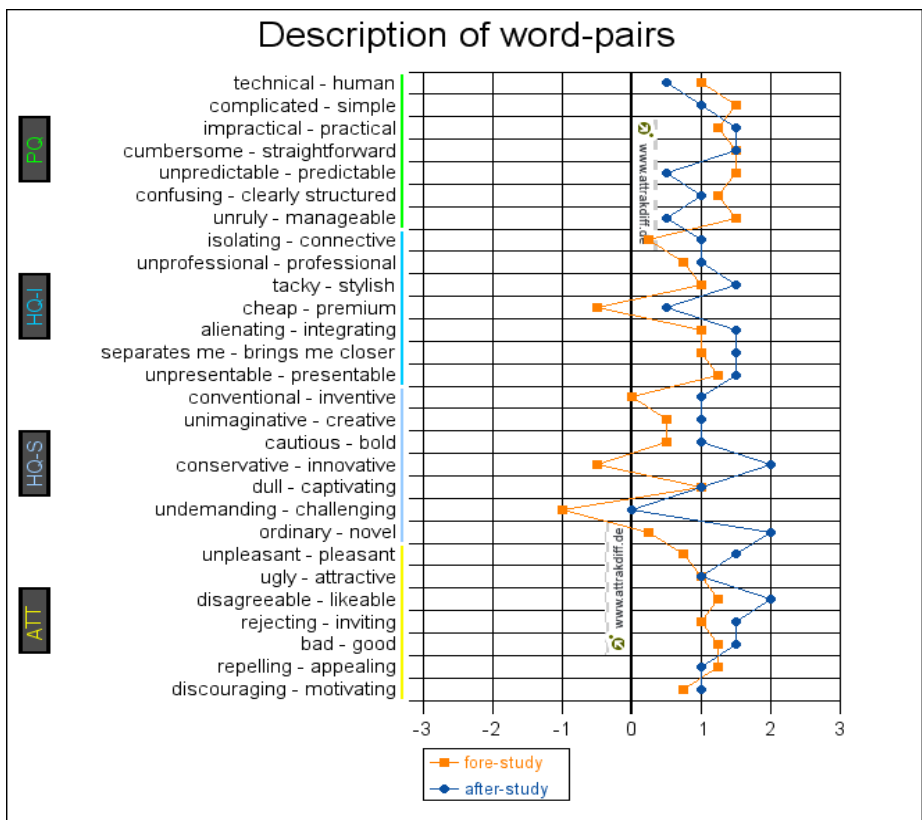

Fig. 6. Mean Values of word pairs for the results in fore-study ("without tactile") and after-study ("with tactile").

\subsection{Significance of the results obtained}

The experiment concludes that the violinists prefer vibrotactile perception synchronous with the notes played than no vibration condition. This is clearly reflected in the Fig. 4. 
In terms of pragmatic quality the product has declined. In terms of hedonic quality the product has improved. The hedonic quality is better than the fore-study. The difference between the pragmatic quality between the fore and after study is statistically insignificant. Similarly, in case of the hedonic quality the results are statistically less insignificant (shown in Fig. 5 and Fig. 6).

\section{Conclusion}

Vibrations transferred from instrument to performer, however, are usually missing when performing with electronic instruments, since the user interface is typically separated from the sound production machinery and thus does not vibrate acoustically. Hence, it is really important to introduce audio tactile vibration feedback synchronous with the instrument, for better performance.

The project examined the use of vibrotactile feedback in an electronic musical instrument and its effect on the instrument from the performer's perspective. By integrating loudspeakers and amplifiers in to the setup of the Violin, we produced an instrument that mimics the vibrotactile feedback found in acoustic instruments. That is, the sound production also produces the vibrotactile feedback [1].This study analyses the influence of the audio induced vibrations on the perceived quality of the experience. Therefore, sound and vibrations must be synchronous because the correlation between the sound and vibration must be naturally strong. Different parameter during this processes (algorithms) are examined in relation to their perceptual consequences using psychophysical experiments. It can be concluded that vibrations play significant role in the perception of music[9].

A number of devices which could be used to simulate the vibration of an acoustic instrument are introduced and implemented. Finally, the hardware setup for the instrument introduces integrated audio speakers to produce both audio and vibrotactile feedback for the performer. This additional feedback to the performer would seem to improve the "feel" of the instrument, so that it is associated more with being an instrument rather than a computer controller.

\section{Acknowledgements}

I would like to express my heart felt gratitude to my mentor, Mr. Sebastian Merchel, TU Dresden, Germany for helping me by giving his valuable suggestions and expertise on the subject. The successful completion of this endeavour is attributed to the work conducted by him in the field without which this paper would not have been possible.

\section{REFERENCES}

[1] Mark T. Marshall, Marcelo M. Wanderley (Input Devices and Musical Interaction Laboratory McGill University - Music Technology), "Vibrotactile Feedback in Digital Musical Instruments" in proceedings of the 2006 International Conference on New Interfaces for Musical Expression (NIME06), Paris, France.

[2] Mark T. Marshall, Marcelo M. Wanderley "Examining the Effects of Embedded Vibrotactile Feedback on the Feel of a Digital Musical Instrument”, Input Devices and Music Interaction Laboratory Centre for Interdisciplinary Research in Music Media and Technology McGill University - Montreal, QC, Canada

[3] M. E. Altinsoy, "Auditory-Tactile Interaction in Virtual Environments" (PhD Thesis, Shaker Verlag) (2006). N. Armstrong. “An Enactive Approach to Digital Musical Instrument Design.” PhD thesis, Princeton University, Nov. 2006.

[4] M. E. Altinsoy, "Auditory-Tactile Interaction in Virtual Environments" (PhD Thesis, Shaker Verlag) (2006).

[5] Wollmana, C. Fritza and J. Frelatb, "Vibrotactile feedback in the left hand of violinists", in proceedings of the Acoustics 2012 Nantes Conference 23-27 April 2012, Nantes, France 3605.

[6] Sebastian Merchel and M. Ercan Altinsoy "Vibration in Music Perception" Presented the Audio Engineering Society, 134th Convention 2013 May 4-7, Rome, Italy

[7] Joseph Malloch, "Adding vibrotactile feedback to the T-stick digital musical instrument" Input Devices and Music Interaction Laboratory Centre for Interdisciplinary Research in Music Media and Technology McGill University - Montreal, QC, Canada

[8] Sebastian Merchel, M. Ercan Altinsoy and Maik Stamm, “Touch the Sound: Audio-Driven Tactile Feedback for Audio Mixing Applications", Chair of Communication Acoustics, Dresden University of Technology, Germany

[9] C. Chafe." Tactile audio feedback.” In Proceedings of the 1993 International Computer Music Conference (ICMC93), pages 76 79, 1993.Journal / Conference Papers.

[10] D. Birnbaum and M. M.Wanderley. "A systematic approach to musical vibrotactile feedback." In Proceedings of the International Computer Music Conference '07 (ICMC'07), volume 2, page 397, Copenhagen, Denmark, 2007.

[11] B. Bongers. "The use of active tactile and force feedback in timbre controlling electronic instruments." In Proceedings of the 1994 International Computer Music Conference, pages 171-174, Arhus, Denmark, 1994.

[12] A. Askenfelt and E. Jansson. "On vibration sensation and finger touch in stringed instrument playing. Music Perception," 9(3):311\{350, 1992 . 